\title{
Effects of Mutual Coupling and Noise Correlation on Downlink Coordinated Beamforming with Limited Feedback
}

\author{
Yuhan Dong, Carlo P. Domizioli, and Brian L. Hughes \\ Department of Electrical and Computer Engineering, North Carolina State University, Raleigh, NC 27695, USA \\ Correspondence should be addressed to Yuhan Dong, ydong@ncsu.edu
}

Received 1 December 2008; Revised 27 March 2009; Accepted 22 April 2009

Recommended by Markus Rupp

\begin{abstract}
We consider the impact of receiver correlation, antenna coupling, matching, and noise on the performance of coordinated beamforming systems. We present a new coordinated beamforming technique for two receivers that is suitable for MIMO broadcast channels with signal and noise correlation at the receiver. We then apply this technique to the specific type of signal and noise correlation that occurs in the presence of receiver mutual coupling. Numerical results suggest that, even in the presence of strong coupling, most of the benefits of coordinated beamforming can be preserved by using appropriate matching networks and linear beamforming. Moreover, these benefits can be achieved even when feedback is limited.
\end{abstract}

Copyright (C) 2009 Yuhan Dong et al. This is an open access article distributed under the Creative Commons Attribution License, which permits unrestricted use, distribution, and reproduction in any medium, provided the original work is properly cited.

\section{Introduction}

In recent years, considerable attention has been paid to multiple-input multiple-output (MIMO) systems, which use multiple antennas at the transmitter and receiver to improve the performance of wireless communication systems over fading multipath channels [1]. In a broadcast setting, when channel state information (CSI) is available at the transmitter, linear beamforming techniques can be a simple and efficient way to communicate data from a base station to multiple mobile receivers [2].

A wide variety of linear beamforming techniques for multiuser MIMO channels have been proposed in the literature. Most of these techniques seek to suppress multiuser interference using perfect CSI at the transmitter [2-5]. If the channel is not reciprocal and the capacity of the feedback link is limited, however, it may be difficult for the transmitter to obtain perfect CSI. As a consequence, several recent works have considered the problem of coordinated beamforming in the presence of limited channel state feedback [6-8].

Most prior work on coordinated beamforming has assumed that the receive antennas are spaced sufficiently far apart so that signal fading and noise can be modeled as independent in each receiver chain. However, many wireless devices, such as cellular handsets and wireless LAN cards, are severely limited in physical size. When multiple antennas are packed into a small space, strong interactions can occur among the antenna elements: the electric fields detected by different elements become correlated, the radiation patterns may become distorted, mutual coupling occurs between the antennas [9-12], and the noise may no longer be spatially white $[13,14]$. Moreover, the statistics of the signal and noise will depend in general on detailed aspects of the receiver design, such as the antenna impedances, matching networks, and amplifiers employed in the receiver RF front-ends. To optimize performance in such scenarios, it is necessary to develop realistic models of these interactions as well as new beamforming techniques that exploit these models to improve performance.

In this paper, we investigate the effects of receive antenna coupling, matching networks and correlated noise on the design and performance of downlink coordinated beamforming systems. We present a new coordinated beamforming technique for two receivers that is suitable for MIMO broadcast channels with signal and noise correlation at the receiver. We then apply this technique to the specific type of signal and noise correlation that occurs in the presence of receiver mutual coupling. Numerical results suggest that, even in the presence of strong coupling, most of the benefits of coordinated beamforming can be preserved by using 


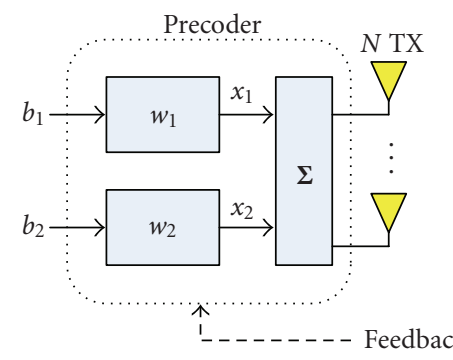

Base station

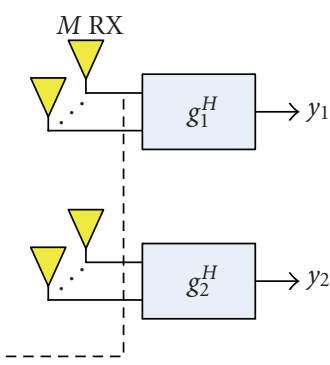

Mobile stations
FIGURE 1: A MIMO broadcast channel with coordinated beamforming.

appropriate matching networks and linear beamforming. Moreover, these benefits can be achieved even when feedback is limited.

The rest of the paper is organized as follows. In Section 2, we present a new coordinated beamforming technique for channels with signal and noise correlation at the receivers. In Section 3, we present a model for a multiantenna receiver front-end that characterizes the signal and noise correlation that results from mutual coupling. Finally, in Section 4, we present numerical results to illustrate the performance of the proposed beamforming technique and its dependence on the properties of the receive array, matching networks, amplifiers, and channel state feedback.

\section{Coordinated Beamforming with Correlated Noise}

When receive antennas are placed close together, the signal components in each receiver chain may become correlated. In a similar way, recent results have shown that mutual coupling among the receive antennas can also cause the noise in each chain to become correlated (e.g., $[13,14])$. In this section, we present a coordinated beamforming strategy suitable for MIMO broadcast channels with signal and noise correlation at the receiver. The specific form of signal and noise correlation that results from mutual coupling is presented in the next section.

Consider a MIMO broadcast channel in which a base station with $N$ transmit antennas sends data to two users with $M$ receive antennas each, as shown in Figure 1. It is well known that the sum-rate capacity of this channel is achieved by "dirty paper" coding, which can be difficult to implement in practice. We will therefore consider the simpler (but suboptimal) transmit beamforming scheme studied in $[5,8]$. In this scheme, the base station transmits one symbol to each user via linear beamforming, so that $\mathbf{x}=b_{1} \mathbf{w}_{1}+b_{2} \mathbf{w}_{2}$ is transmitted, where $b_{k}$ is the symbol intended for the $k$ th user and $\mathbf{w}_{k}$ is a unit-norm beamformer. We assume a rich scattering environment with a delay spread that is small compared to the inverse signal bandwidth, so the complex baseband signal detected at the $k$-th user may be expressed as

$$
\mathbf{r}_{k}=\mathbf{H}_{k} \mathbf{x}+\mathbf{n}_{k}
$$

where $\mathbf{H}_{k}$ is the $M \times N$ channel matrix between the transmitter and the $k$-th user, and $\mathbf{n}_{k}$ represents noise. (These results extend in a natural way to frequency-selective channels with OFDM, but we will not consider this here.) We assume the $N$ transmit antennas are spaced far enough apart so as to be essentially uncoupled and uncorrelated, so the columns of $\mathbf{H}_{k}$ are independent zero-mean circularlysymmetric complex Gaussian vectors with covariance $\mathbf{S}_{k}$. Hereafter we denote this distribution by $\mathcal{C} \mathcal{N}\left(\mathbf{0}, \mathbf{S}_{k}\right)$. The noise is also modeled as Gaussian, $\mathbf{n}_{k} \sim \mathcal{C} \mathcal{N}\left(\mathbf{0}, \mathbf{T}_{k}\right)$. Expressions for $\boldsymbol{S}_{k}$ and $\mathbf{T}_{k}$ are derived in Section 3.

The $k$-th user applies a unit-norm linear combiner $\mathbf{g}_{k}$ to the received signal to form the decision statistics:

$$
\begin{aligned}
& y_{1}=\mathbf{g}_{1}^{H} \mathbf{r}_{1}=\mathbf{g}_{1}^{H} \mathbf{H}_{1} \mathbf{w}_{1} b_{1}+\mathbf{g}_{1}^{H} \mathbf{H}_{1} \mathbf{w}_{2} b_{2}+\mathbf{g}_{1}^{H} \mathbf{n}_{1}, \\
& y_{2}=\mathbf{g}_{2}^{H} \mathbf{r}_{2}=\mathbf{g}_{2}^{H} \mathbf{H}_{2} \mathbf{w}_{1} b_{1}+\mathbf{g}_{2}^{H} \mathbf{H}_{2} \mathbf{w}_{2} b_{2}+\mathbf{g}_{2}^{H} \mathbf{n}_{2},
\end{aligned}
$$

where the superscript $H$ denotes the conjugate-transpose.

We want to design beamformers $\mathbf{w}_{1}, \mathbf{w}_{2}$ and combiners $\mathbf{g}_{1}, \mathbf{g}_{2}$ so as to maximize the sum-rate of this system, subject to the constraint that no interference is present in either decision statistic. The zero interference constraint implies

$$
\mathbf{g}_{1}^{H} \mathbf{H}_{1} \mathbf{w}_{2}=\mathbf{g}_{2}^{H} \mathbf{H}_{2} \mathbf{w}_{1}=0,
$$

in which case the sum-rate of the resulting system is given by

$$
C\left(\mathbf{w}_{1}, \mathbf{w}_{2}, \mathbf{g}_{1}, \mathbf{g}_{2}\right)=\log _{2}\left(1+\gamma_{1}\right)+\log _{2}\left(1+\gamma_{2}\right),
$$

where $P_{k}=E\left[\left|b_{k}\right|^{2}\right]$ is the transmit power allotted to user $k$ and

$$
\gamma_{k}=\frac{P_{k}\left|\mathbf{g}_{k}^{H} \mathbf{H}_{k} \mathbf{w}_{k}\right|^{2}}{\mathbf{g}_{k}^{H} \mathbf{T}_{k} \mathbf{g}_{k}}
$$

is the output signal-to-noise ratio (SNR) of the $k$-th receiver. We assume full channel state information is available, so $\mathbf{H}_{1}$ and $\mathbf{H}_{2}$ are known at the transmitter; the case of limited feedback is considered below. The optimization problem is therefore to maximize (4) over all unit-norm vectors $\mathbf{w}_{1}, \mathbf{w}_{2}, \mathbf{g}_{1}, \mathbf{g}_{2}$ that satisfy (3). Note that we can relax the assumption that $\mathbf{g}_{1}$ and $\mathbf{g}_{2}$ have unit norm, since (4) does not depend on the norms of the combiners.

Since the design of the beamformers $\mathbf{w}_{1}$ and $\mathbf{w}_{2}$ involves coordination of the two users, this problem has been called coordinated beamforming [8].

2.1. Uncorrelated Fading and Noise. For uncorrelated fading $\left(\mathbf{S}_{1}=\mathbf{S}_{2}=\mathbf{I}\right)$ and spatially white noise $\left(\mathbf{T}_{1}=\mathbf{T}_{2}=N_{0} \mathbf{I}\right)$, this optimization problem has been studied in $[5,8]$. For $M \geq$ $N=2$, Wong [5] asserts that the optimal receiver processing is maximal-ratio combining (MRC),

$$
\mathbf{g}_{1}=\mathbf{H}_{1} \mathbf{w}_{1}, \quad \mathbf{g}_{2}=\mathbf{H}_{2} \mathbf{w}_{2}
$$

in which case the zero interference constraint (3) becomes

$$
\mathbf{w}_{1}^{H} \mathbf{H}_{1}^{H} \mathbf{H}_{1} \mathbf{w}_{2}=\mathbf{w}_{2}^{H} \mathbf{H}_{2}^{H} \mathbf{H}_{2} \mathbf{w}_{1}=0 .
$$


Assuming MRC is used at the receiver, Chae et al. [8] showed for $M \geq N \geq 2$ that any beamformers $\mathbf{w}_{1}$ and $\mathbf{w}_{2}$ that satisfy (7) must be generalized eigenvectors of the matrices

$$
\mathbf{F}_{1}=\mathbf{H}_{1}^{H} \mathbf{H}_{1}, \quad \mathbf{F}_{2}=\mathbf{H}_{2}^{H} \mathbf{H}_{2},
$$

or equivalently, eigenvectors of the matrix $\mathbf{F}_{2}^{-1} \mathbf{F}_{1}$ if $\mathbf{F}_{2}$ is nonsingular. (The optimal beamformers in [8] are expressed in a slightly different form, as generalized eigenvectors of the normalized matrices $\overline{\mathbf{F}}_{1}=\mathbf{F}_{1} / \operatorname{Tr}\left[\mathbf{F}_{1}\right]$ and $\overline{\mathbf{F}}_{2}=\mathbf{F}_{2} / \operatorname{Tr}\left[\mathbf{F}_{2}\right]$, where $\operatorname{Tr}[\cdot]$ denotes the trace. Clearly, the generalized eigenvectors of $\overline{\mathbf{F}}_{1}$ and $\overline{\mathbf{F}}_{2}$ are the same as those of $\mathbf{F}_{1}$ and $\mathbf{F}_{2}$.)

Based on this result, Chae et al. proposed the following approach to coordinated beamforming. First we compute a set $\mathbf{W}$ of generalized eigenvectors of $\mathbf{F}_{1}$ and $\mathbf{F}_{2}$. We then choose beamformers from this set so as to maximize the sum-rate when MRC combining is used:

$$
\left\{\mathbf{w}_{1}^{o}, \mathbf{w}_{2}^{o}\right\}=\arg \max _{\mathbf{w}_{1}, \mathbf{w}_{2} \in \mathbf{W}, \mathbf{w}_{1} \neq \mathbf{w}_{2}} C\left(\mathbf{w}_{1}, \mathbf{w}_{2}, \mathbf{H}_{1} \mathbf{w}_{1}, \mathbf{H}_{2} \mathbf{w}_{2}\right) .
$$

Simulations results presented in [8] suggest that this method can achieve a sum-rate close to the sum-capacity of the MIMO broadcast channel.

2.2. Correlated Fading and Noise. When signal fading is correlated $\left(\mathbf{S}_{1} \neq \mathbf{I}\right.$ or $\left.\mathbf{S}_{2} \neq \mathbf{I}\right)$ but the noise is spatially white, the coordinated beamforming strategy in [8] can still be applied as written, although the resulting average sum-rate will naturally depend on the covariances $\boldsymbol{S}_{1}, \boldsymbol{S}_{2}$. The situation is more complicated when channel noise is correlated. When $\mathbf{T}_{1} \neq N_{0} \mathbf{I}$ or $\mathbf{T}_{2} \neq N_{0} \mathbf{I}$, the strategy in [8] still eliminates multiuser interference in the decision statistics (2), but does not generally provide the best sum-rate because it contains no preference for beamformers aligned to directions with minimum channel noise.

To develop a strategy suitable for correlated noise, consider a channel with nonsingular noise covariances $\mathbf{T}_{1}$ and $\mathbf{T}_{2}$. Let $\mathbf{T}_{k}^{1 / 2}$ denote the Hermitian square-root of $\mathbf{T}_{k}$. Substituting the change of variables

$$
\mathbf{g}_{1}=\mathbf{T}_{1}^{-1 / 2} \mathbf{u}_{1}, \quad \mathbf{g}_{2}=\mathbf{T}_{2}^{-1 / 2} \mathbf{u}_{2}
$$

into (2), we obtain the decision statistics

$$
\begin{aligned}
& y_{1}=\mathbf{u}_{1}^{H} \mathbf{T}_{1}^{-1 / 2} \mathbf{H}_{1} \mathbf{w}_{1} b_{1}+\mathbf{u}_{1}^{H} \mathbf{T}_{1}^{-1 / 2} \mathbf{H}_{1} \mathbf{w}_{2} b_{2}+\mathbf{u}_{1}^{H} \mathbf{T}_{1}^{-1 / 2} \mathbf{n}_{1}, \\
& y_{2}=\mathbf{u}_{2}^{H} \mathbf{T}_{2}^{-1 / 2} \mathbf{H}_{2} \mathbf{w}_{1} b_{1}+\mathbf{u}_{2}^{H} \mathbf{T}_{2}^{-1 / 2} \mathbf{H}_{2} \mathbf{w}_{2} b_{2}+\mathbf{u}_{2}^{H} \mathbf{T}_{2}^{-1 / 2} \mathbf{n}_{2} .
\end{aligned}
$$

Observe that choosing vectors $\mathbf{w}_{1}, \mathbf{w}_{2}, \mathbf{u}_{1}, \mathbf{u}_{2}$ to eliminate multiuser interference and to optimize the resulting sumrate of the channel (11) is mathematically equivalent to the original optimization problem (2) with $\mathbf{H}_{1}, \mathbf{H}_{2}, \mathbf{n}_{1}$, and $\mathbf{n}_{2}$ replaced by $\hat{\mathbf{H}}_{1}=\mathbf{T}_{1}^{-1 / 2} \mathbf{H}_{1}, \hat{\mathbf{H}}_{2}=\mathbf{T}_{2}^{-1 / 2} \mathbf{H}_{2}, \widehat{\mathbf{n}}_{1}=\mathbf{T}_{1}^{-1 / 2} \mathbf{n}_{1}$ and $\hat{\mathbf{n}}_{2}=\mathbf{T}_{2}^{-1 / 2} \mathbf{n}_{2}$, respectively.
Since the new noise vectors $\widehat{\mathbf{n}}_{1}$ and $\widehat{\mathbf{n}}_{2}$ are both $\mathcal{C} \mathcal{N}(\mathbf{0}, \mathbf{I})$, we can now apply the beamforming strategy for uncorrelated noise in [8]. If MRC combining is used, then $\mathbf{u}_{1}=\hat{\mathbf{H}}_{1} \mathbf{w}_{1}$ and $\mathbf{u}_{2}=\hat{\mathbf{H}}_{2} \mathbf{w}_{2}$, or equivalently

$$
\mathbf{g}_{1}=\mathbf{T}_{1}^{-1} \mathbf{H}_{1} \mathbf{w}_{1}, \quad \mathbf{g}_{2}=\mathbf{T}_{2}^{-1} \mathbf{H}_{2} \mathbf{w}_{2} .
$$

For these combiners, the zero interference constraint (3) becomes

$$
\mathbf{w}_{1}^{H} \mathbf{H}_{1}^{H} \mathbf{T}_{1}^{-1} \mathbf{H}_{1} \mathbf{w}_{2}=\mathbf{w}_{2}^{H} \mathbf{H}_{2}^{H} \mathbf{T}_{2}^{-1} \mathbf{H}_{2} \mathbf{w}_{1}=0 .
$$

For any $M \geq N \geq 2$, we conclude that any beamformers $\mathbf{w}_{1}$ and $\mathbf{w}_{2}$ that satisfy (13) must be generalized eigenvectors of the matrices

$$
\widehat{\mathbf{F}}_{1}=\mathbf{H}_{1}^{H} \mathbf{T}_{1}^{-1} \mathbf{H}_{1}, \quad \widehat{\mathbf{F}}_{2}=\mathbf{H}_{2}^{H} \mathbf{T}_{2}^{-1} \mathbf{H}_{2} .
$$

We can now extend the coordinated beamforming strategy in [8] to correlated fading and noise. First compute a set $\widehat{\mathbf{W}}$ of generalized eigenvectors of $\widehat{\mathbf{F}}_{1}$ and $\widehat{\mathbf{F}}_{2}$. If the combiners in (12) are used, then the sum-rate is maximized by choosing the beamformers as follows:

$\left\{\widehat{\mathbf{w}}_{1}^{o}, \widehat{\mathbf{w}}_{2}^{o}\right\}=\arg \max _{\mathbf{w}_{1}, \mathbf{w}_{2} \in \widehat{\mathbf{W}}, \mathbf{w}_{1} \neq \mathbf{w}_{2}} C\left(\mathbf{w}_{1}, \mathbf{w}_{2}, \mathbf{T}_{1}^{-1} \mathbf{H}_{1} \mathbf{w}_{1}, \mathbf{T}_{2}^{-1} \mathbf{H}_{2} \mathbf{w}_{2}\right)$.

Simulations of the performance of this strategy for receiver mutual coupling are given in Section 4 . We conclude this section with a general observation about the average performance of this beamforming strategy.

Theorem 1. The expected sum-rate of the coordinated beamforming strategy above,

$$
\bar{C}=E\left[\max _{\mathbf{w}_{1}, \mathbf{w}_{2} \in \widehat{\mathbf{W}}, \mathbf{w}_{1} \neq \mathbf{w}_{2}} C\left(\mathbf{w}_{1}, \mathbf{w}_{2}, \mathbf{T}_{1}^{-1} \mathbf{H}_{1} \mathbf{w}_{1}, \mathbf{T}_{2}^{-1} \mathbf{H}_{2} \mathbf{w}_{2}\right)\right],
$$

depends on $\mathbf{S}_{1}, \mathbf{S}_{2}, \mathbf{T}_{1}, \mathbf{T}_{2}$ only through the eigenvalues of the "SNR" matrices $\mathbf{S}_{1} \mathbf{T}_{1}^{-1}$ and $\mathbf{S}_{2} \mathbf{T}_{2}^{-1}$.

Proof of Theorem 1. From (4) and (5), observe that for $\mathbf{w}_{1}, \mathbf{w}_{2} \in \widehat{\mathbf{W}}, \mathbf{w}_{1} \neq \mathbf{w}_{2}$

$$
\begin{aligned}
C\left(\mathbf{w}_{1}, \mathbf{w}_{2}, \mathbf{T}_{1}^{-1} \mathbf{H}_{1} \mathbf{w}_{1}, \mathbf{T}_{2}^{-1} \mathbf{H}_{2} \mathbf{w}_{2}\right) \\
\quad=\log _{2}\left(1+P_{1} \mathbf{w}_{1}^{H} \hat{\mathbf{F}}_{1} \mathbf{w}_{1}\right)+\log _{2}\left(1+P_{2} \mathbf{w}_{2}^{H} \hat{\mathbf{F}}_{2} \mathbf{w}_{2}\right) .
\end{aligned}
$$

so $\bar{C}$ depends only on the distributions of the independent random matrices $\hat{\mathbf{F}}_{1}$ and $\hat{\mathbf{F}}_{2}$ in (14). The first channel matrix can be written as $\mathbf{H}_{1}=\mathbf{S}_{1}^{1 / 2} \tilde{\mathbf{H}}_{1}$, where $\widetilde{\mathbf{H}}_{1}$ is a matrix with independent $\mathcal{C} \mathcal{N}(0,1)$ entries, and thus $\widehat{\mathbf{F}}_{1}=$ $\tilde{\mathbf{H}}_{1}^{H} \mathbf{S}_{1}^{1 / 2} \mathbf{T}_{1}^{-1} \mathbf{S}_{1}^{1 / 2} \tilde{\mathbf{H}}_{1}$. The eigenvalue decomposition yields

$$
\mathbf{S}_{1}^{1 / 2} \mathbf{T}_{1}^{-1} \mathbf{S}_{1}^{1 / 2}=\mathbf{U}^{H} \boldsymbol{\Lambda}_{1} \mathbf{U}
$$

where $\Lambda_{1}$ is a diagonal eigenvalue matrix and $\mathbf{U}$ is an $M \times M$ unitary matrix. Since $\overline{\mathbf{H}}_{1}=\mathbf{U} \tilde{\mathbf{H}}_{1}$ and $\tilde{\mathbf{H}}_{1}$ have the 
same probability distribution, it follows that $\widehat{\mathbf{F}}_{1}=\overline{\mathbf{H}}_{1}^{H} \boldsymbol{\Lambda}_{1} \overline{\mathbf{H}}_{1}$ where $\overline{\mathbf{H}}_{1}$ is a matrix with independent $\mathcal{C} \mathcal{N}(0,1)$ entries. Since $\mathbf{S}_{1}^{1 / 2} \mathbf{T}_{1}^{-1} \mathbf{S}_{1}^{1 / 2}$ and $\mathbf{S}_{1} \mathbf{T}_{1}^{-1}$ have the same eigenvalues, the distribution of $\hat{\mathbf{F}}_{1}$ thus depends only on the eigenvalues of $\mathbf{S}_{1} \mathbf{T}_{1}^{-1}$. Proceeding in the same way, we can show that the distribution of $\hat{\mathbf{F}}_{2}$ depends only on the eigenvalues of $\mathbf{S}_{2} \mathbf{T}_{2}^{-1}$, which completes the proof.

2.3. Limited Feedback. The coordinated beamforming scheme in Section 2.2 requires the matrices $\widehat{\mathbf{F}}_{1}$ and $\widehat{\mathbf{F}}_{2}$ to be fed back by the users to the base station. When feedback is limited, the base station's estimates of these matrices may be imprecise and the resulting performance degraded. In this paper, we also examine the impact of mutual coupling and noise correlation on the performance of coordinated beamforming with limited feedback. In particular, we consider a scenario in which no CSI is available at the base station but there exists a low-rate, error-free, zero-delay feedback link. In simulations and analyses, we adopt the simple limited feedback method proposed in [8], in which the entries of the normalized matrices

$$
\mathbf{G}_{k}=\frac{\widehat{\mathbf{F}}_{k}}{\operatorname{Tr}\left[\widehat{\mathbf{F}}_{k}\right]}, \quad k=1,2
$$

are uniformly quantized and fed back to the transmitter. As shown in [8], these matrices are Hermitian, preserve the generalized eigenvectors, and the entries have welldefined ranges. For example, for $N=2$ we have $\left[\mathbf{G}_{k}\right]_{11} \in$ $[0,1],\left[\mathbf{G}_{k}\right]_{22}=1-\left[\mathbf{G}_{k}\right]_{11}$ and $\operatorname{Re}\left\{\left[\mathbf{G}_{k}\right]_{12}\right\}, \operatorname{Im}\left\{\left[\mathbf{G}_{k}\right]_{12}\right\} \in$ $[-0.5,0.5]$. To quantize all of the real scalars in these matrices using $Q$ bits requires a total of $\left(N^{2}-1\right) Q$ bits for each user.

When beamformers (15) are designed using quantized versions of the channel matrices $\mathbf{H}_{1}$ and $\mathbf{H}_{2}$, the multiuser interference in the decision statistics (2) may not be completely canceled. As a consequence, to evaluate the performance of limited-feedback beamformers, we must replace the SNRs (5) in the sum-rate (4) with the signal-tointerference-and-noise ratios (SINRs)

$$
\gamma_{k}=\frac{P_{k}\left|\mathbf{g}_{k}^{H} \mathbf{H}_{k} \mathbf{w}_{k}\right|^{2}}{P_{l}\left|\mathbf{g}_{k}^{H} \mathbf{H}_{k} \mathbf{w}_{l}\right|^{2}+\mathbf{g}_{k}^{H} \mathbf{T}_{k} \mathbf{g}_{k}},
$$

where $l=1$ if $k=2$ and $l=2$ when $k=1$.

\section{Receiver Model}

We now present a model for a multi-antenna receiver with correlation and mutual coupling. The aim is to derive physical expressions for the correlation matrices $S_{1}, S_{2}, T_{1}, T_{2}$ introduced in Section 2.2. Since the mechanisms that lead to correlation in both receivers are similar, throughout this section we focus solely on receiver 1 .

We consider the circuit model for an $M$-antenna receiver introduced in [14], which is illustrated in Figure 2. This model includes the impedances of the antenna array, matching network, front-end amplifiers and load, as well as the currents and voltages due to the signal and dominant noise sources. (For simplicity, the downstream noise in $[14,(25)]$ is omitted, so $r_{d}=0$.) We assume the system is narrowband, so that all impedances are constant over the system bandwidth and all signals can be expressed in complex baseband form.

Antenna Array. The antenna array can be represented as an $M$-port Thevenin equivalent circuit, as shown in Figure 2. The relationship between terminal voltages and currents is then described by an $M \times M$ impedance matrix $\mathbf{Z}_{A}$, where $\left[\mathbf{Z}_{A}\right]_{m m}$ is the self-impedance of antenna $m$, and $\left[\mathbf{Z}_{A}\right]_{m n}$ is the mutual impedance between antennas $n$ and $m$. For a uniform linear array of $M$ thin dipoles, approximate formulas for these impedances are given in $[15,(8-71)]$. These impedances can also be estimated by numerical techniques (more on this in Section 4).

The antenna array converts the incident electromagnetic field into an open-circuit voltage $\mathbf{V}_{o}$ across the antenna terminals. Since this voltage contains both signal and noise components, it can be written as

$$
\mathbf{V}_{o}=\mathbf{H}_{o} \mathbf{x}+\mathbf{n}_{o},
$$

where $\mathbf{H}_{o} \mathbf{X}$ is the voltage induced by the transmitted signal $\mathbf{x}$ and $\mathbf{n}_{o}$ represents noise. We assume that the columns of $\mathbf{H}_{o}$ are independent, $\mathcal{C} \mathcal{N}\left(\mathbf{0}, \boldsymbol{S}_{o}\right)$ random vectors. For a uniform linear array, some authors [9-11] have modeled the opencircuit signal covariance by Clarke's model

$$
\left[\mathbf{S}_{o}\right]_{m n}=J_{0}\left(\frac{2 \pi d|m-n|}{\lambda}\right),
$$

where $d$ is the interelement spacing, $\lambda$ is the wavelength, and $J_{0}$ is the zeroth-order Bessel function of the first kind. Simulations suggest that this model may not be accurate for small $d$, however, so we estimate this matrix numerically in Section 4 .

For perfectly conducting antennas, $\mathbf{n}_{o}$ represents the voltage induced in the array by noise from the surrounding environment. As in [14], we consider here thermal noise from an isotropic distribution of black-body radiators with a uniform temperature $T_{0}$. In this case, the noise voltage is $\mathbf{n}_{o} \sim \mathcal{C} \mathcal{N}\left(\mathbf{0}, \mathbf{T}_{o}\right)$, where [14]

$$
\mathbf{T}_{o}=2 k_{B} T_{0} B\left(\mathbf{Z}_{A}+\mathbf{Z}_{A}^{H}\right),
$$

$k_{B}=1.38 \times 10^{-23} \mathrm{~J} / \mathrm{K}$ is Boltzmann's constant and $B$ is the system bandwidth in Hz. In this paper, we take $T_{0}=290$ $\mathrm{K}$, the standard temperature. Note that, in the absence of mutual coupling, $\mathbf{Z}_{A}$ is diagonal and the noise is spatially white. When coupling is present, however, $\mathbf{Z}_{A}$ is no longer diagonal and the noise is correlated.

Matching. A matching network is often used to alter the antenna array impedance, usually in order to maximize the power transfer or minimize the noise factor of the amplifiers. This network is usually formed from passive, reactive elements so it is noiseless, lossless, and reciprocal. If $\mathbf{V}_{1}, \mathbf{I}_{1}, \mathbf{V}_{2}, \mathbf{I}_{2}$ denote the voltages and currents at the network 


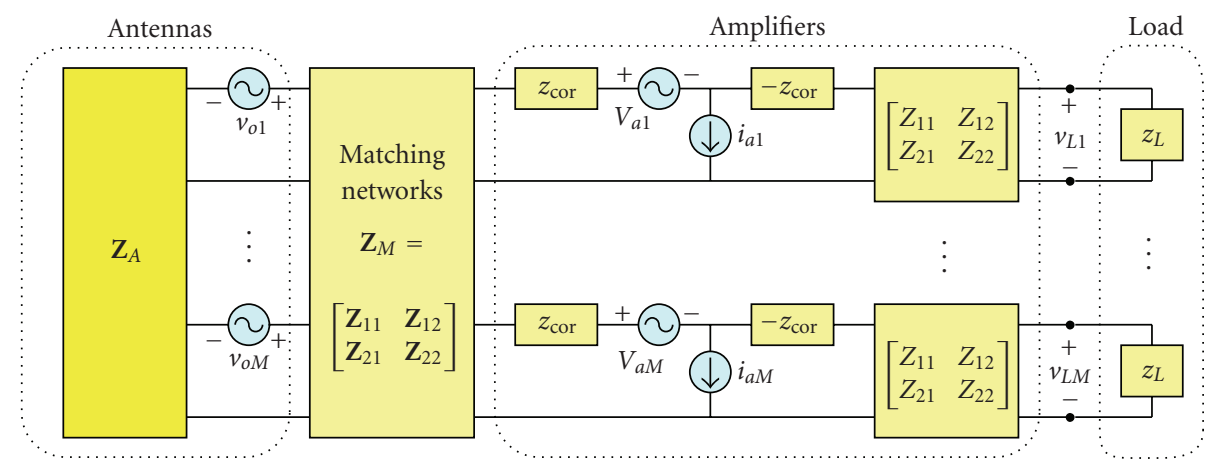

FIgURE 2: Circuit model of an $M$-antenna receiver with mutual coupling.

input and output, respectively, then the network is described by four $M \times M$ impedance matrices:

$$
\begin{aligned}
& \mathbf{V}_{1}=\mathbf{Z}_{11} \mathbf{I}_{1}+\mathbf{Z}_{12} \mathbf{I}_{2}, \\
& \mathbf{V}_{2}=\mathbf{Z}_{21} \mathbf{I}_{1}+\mathbf{Z}_{22} \mathbf{I}_{2} .
\end{aligned}
$$

As shown in $[16,(2.4)-(2.5)]$, this network is lossless (i.e., dissipates no power) if the following conditions are satisfied: $\mathbf{Z}_{11}=-\mathbf{Z}_{11}^{H}, \mathbf{Z}_{12}=-\mathbf{Z}_{21}^{H}$, and $\mathbf{Z}_{22}=-\mathbf{Z}_{22}^{H}$. The antennas and matching network together constitute a noisy linear network which can be represented as a Thevenin equivalent circuit with open-circuit voltage [16, page 13]

$$
\mathbf{V}_{o}^{\prime}=\mathbf{M V}_{o}, \quad \mathbf{M}=\mathbf{Z}_{21}\left(\mathbf{Z}_{11}+\mathbf{Z}_{A}\right)^{-1},
$$

and impedance

$$
\mathbf{Z}_{A}^{\prime}=\mathbf{Z}_{22}-\mathbf{M} \mathbf{Z}_{12}
$$

These equations will be applied to some specific matching networks of interest in Section 4.

Amplifiers. The matching network is connected to the load through a bank of $M$ identical, uncoupled amplifiers. Each amplifier can be modeled as a linear, noisy two-port network [14], as shown in Figure 2. The internal noise in amplifier $k$ is represented by a random noise voltage $v_{a k} \sim$ $\mathcal{C} \mathcal{N}\left(0,4 k_{B} T_{0} B r_{a}\right)$ and an independent noise current $i_{a k} \sim$ $\mathcal{C} \mathcal{N}\left(0,4 k_{B} T_{0} B g_{a}\right)$, where $r_{a}$ and $g_{a}$ are the equivalent noise resistance and equivalent noise conductance, respectively. The parameter $z_{\text {cor }}=r_{\text {cor }}+j x_{\text {cor }}$ is called the correlation impedance and models the degree of correlation between the noise observed at the two ports of the amplifier. Note that the noise statistics of an amplifier are completely characterized by the parameters $\left\{r_{a}, g_{a}, z_{\text {cor }}\right\}$.

When an isolated amplifier is connected to a source of impedance $z_{s}=r_{s}+j x_{s}$, the noise factor is defined as the total noise power observed at the output port divided by the noise power contributed by the source alone, which is given by [14]

$$
F=1+\frac{1}{r_{s}}\left(r_{a}+g_{a}\left|z_{s}+z_{\mathrm{cor}}\right|^{2}\right)
$$

The noise factor is a useful metric because it relates the input and output SNRs of the amplifier. In decibels, this relationship is given by $\mathrm{SNR}_{\text {out }}=\mathrm{SNR}_{\text {in }}-\mathrm{NF}$, where $\mathrm{NF}=10 \log _{10} F$ is the noise figure. It can be shown that the minimum value of $F$ is

$$
F_{\min }=1+2\left(g_{a} r_{\mathrm{cor}}+\sqrt{g_{a} r_{a}+\left(g_{a} r_{\mathrm{cor}}\right)^{2}}\right),
$$

which is achieved when $z_{s}=z_{\mathrm{opt}}$ where

$$
z_{\mathrm{opt}}=\sqrt{r_{a} / g_{a}+r_{\mathrm{cor}}^{2}}-j x_{\mathrm{cor}} .
$$

Load. In Section 2, we denoted the input to the first user's combiner by $\mathbf{r}_{1}$ in (1). In our model, $\mathbf{r}_{1}$ is taken to be the voltage $\mathbf{V}_{L}$ observed across the load in Figure 2. We assume that the receiver chains after the amplifiers are uncoupled and each is electrically isolated from the receiver front-end, so each branch of the load can be modeled by an impedance $z_{L}$.

With the assumptions above, the fading covariance in (1) was shown in [14] to be given by

$$
\mathbf{S}_{1}=\mathbf{D C M S}_{o} \mathbf{M}^{H} \mathbf{C}^{H} \mathbf{D}^{H}
$$

where $\boldsymbol{S}_{o}$ is the open-circuit fading covariance (e.g., (22)),

$$
\begin{aligned}
& \mathbf{C}=z_{21}\left(\mathbf{Z}_{A}^{\prime}+z_{11} \mathbf{I}_{M}\right)^{-1}, \\
& \mathbf{D}=z_{L}\left[\left(z_{L}+z_{22}\right) \mathbf{I}_{M}-z_{12} \mathbf{C}\right]^{-1},
\end{aligned}
$$

$\mathbf{I}_{M}$ is the $M \times M$ identity matrix, and $\mathbf{M}$ and $\mathbf{Z}_{A}^{\prime}$ are defined in (25) and (26), respectively. Here $\mathbf{M}$ describes the impact of the matching network on the open-circuit fading covariance, and DC is obtained by using elementary circuit theory to map the fading covariance at the output of the matching network to the corresponding covariance at the load. The noise covariance was shown in $[14,(25)]$ to be

$$
\mathbf{T}_{1}=(\mathbf{D C}) \mathbf{T}_{a}(\mathbf{D C})^{H},
$$

where

$$
\begin{aligned}
\mathbf{T}_{a}=4 k_{B} T_{0} B[ & \frac{1}{2}\left(\mathbf{Z}_{A}^{\prime}+\mathbf{Z}_{A}^{\prime H}\right)+r_{a} \mathbf{I}_{M}+g_{a}\left(\mathbf{Z}_{A}^{\prime}+z_{\mathrm{cor}} \mathbf{I}_{M}\right) \\
& \left.\times\left(\mathbf{Z}_{A}^{\prime}+z_{\mathrm{cor}} \mathbf{I}_{M}\right)^{H}\right],
\end{aligned}
$$


$k_{B}$ is Boltzmann's constant, $T_{0}$ is the noise temperature of the amplifier and surrounding noise (assumed equal), $B$ is the bandwidth, and $\left\{r_{a}, g_{a}, z_{\text {cor }}\right\}$ are the amplifier noise parameters. Intuitively, $\mathbf{T}_{a}$ represents the combined covariance of all noise sources, referred to the output of the matching network.

The covariance $\mathbf{T}_{1}$ and the analogous covariance $\mathbf{T}_{2}$ of receiver 2 are the inputs to the coordinated beamforming strategy proposed in Section 2.2. Note that this strategy in general depends on detailed aspects of the receiver design, such as the antenna impedances, matching networks, amplifier parameters, and surrounding noise environment. The performance of this strategy, however, can be expressed in a somewhat simpler form. From Theorem 1, we know that the average sum-rate performance of this system depends on $S_{1}$ and $T_{1}$ only through the eigenvalues of the SNR matrix $\mathbf{S}_{1} \mathbf{T}_{1}^{-1}$. When DC is non singular, however, then

$$
\mathbf{S}_{1} \mathbf{T}_{1}^{-1}=(\mathbf{D C}) \mathbf{M S} \mathbf{S}_{o}^{H} \mathbf{T}_{a}^{-1}(\mathbf{D C})^{-1}
$$

Since $\mathbf{S}_{1} \mathbf{T}_{1}^{-1}$ is related to $\mathbf{M} \mathbf{S}_{o} \mathbf{M}^{H} \mathbf{T}_{a}^{-1}$ by a similarity transformation, they have the same eigenvalues. From Theorem 1, we can therefore take $\mathbf{S}_{1} \mathbf{T}_{1}^{-1}=\mathbf{M} \mathbf{S}_{o} \mathbf{M}^{H} \mathbf{T}_{a}^{-1}$ for the purposes of evaluating the average sum-rate performance.

\section{Numerical Results}

In this section, we give numerical examples that illustrate how the coordinated beamforming strategy proposed in Section 2.2 performs when applied to mobiles with receiver correlation and mutual coupling, as modeled in Section 3. We consider a system with $N=2$ transmit antennas spaced far enough apart so as to be uncoupled and uncorrelated. We assume the transmitter allocates equal power to each user, so $P_{1}=P_{2}=P / 2$ where $P$ is the total transmit power.

We assume the transmitter sends data to two users with identical receivers. Each receiver employs a uniform linear array of $M=2$ or 4 half-wavelength dipoles with interelement spacing $d$. Each dipole has radius $10^{-3} \lambda$, where $\lambda$ is the signal wavelength.

The open-circuit fading covariance $\boldsymbol{S}_{o}$ was computed as follows. Let $g_{m}(\phi)$ denote the open-circuit voltage induced in the $m$-th receive antenna by a vertically-polarized, unitpower plane wave with angle-of-arrival $\phi$ due to a scatterer located in the antenna far-field. If the received signal consists of a superposition of a large number of plane waves with random phases, which are uniformly distributed in azimuth $\phi$, then the signal is approximately Gaussian with mean zero and covariance $[13,14]$

$$
\left[\mathbf{S}_{o}\right]_{m n}=\frac{1}{2 \pi} \int_{0}^{2 \pi} g_{m}(\phi) g_{n}^{*}(\phi) e^{j 2 \pi(d / \lambda)(m-n) \cos \phi} d \phi .
$$

For omnidirectional antennas $\left(g_{m}(\phi)=1\right)$, this expression reduces to Clarke's model (22). While infinitesimally thin dipoles are often well modeled as omnidirectional, finitediameter dipoles in an array are not. We therefore calculated the functions $g_{m}(\phi)$ using the Numerical Electromagnetics Code (NEC) [17], a program based on the method of moments. NEC was also used to estimate the antenna impedance $Z_{A}$, which should be more accurate than the thin dipole approximations in [15].

We consider the Maxim 2642 SiGe low-noise amplifier [18], which is designed for use in the cellular band. In highgain mode with $R_{\text {bias }}=510 \Omega$ and $f=900 \mathrm{MHz}$, its impedance matrix and noise parameters are given by

$$
\begin{gathered}
{\left[\begin{array}{ll}
z_{11} & z_{12} \\
z_{21} & z_{22}
\end{array}\right]=\left[\begin{array}{cc}
35.7 \angle-82.0^{\circ} & 2.74 \angle 91.8^{\circ} \\
325 \angle-119^{\circ} & 46.1 \angle-23.3^{\circ}
\end{array}\right] \Omega,} \\
r_{a}=9.45 \Omega, \quad g_{a}=3.24 \mathrm{mS}, \quad z_{\text {cor }}=35.3 \angle-114^{\circ} \Omega .
\end{gathered}
$$

To maximize power transfer from the amplifiers to the load, we assume the load is conjugate matched to the amplifier output impedance, so $z_{L}=z_{22}^{*}$.

We consider two matching networks that have been discussed in the literature $[12,13]$. In optimal multiport matching for minimum noise factor, the network $\mathbf{Z}_{M}$ in (26) is chosen so that $\mathbf{Z}_{A}^{\prime}=z_{\text {opt }} \mathbf{I}_{M}$, where $z_{\text {opt }}$ is the source impedance (29) that minimizes the amplifier noise factor. It is easily verified that a lossless reciprocal network that implements this match is

$$
\mathbf{Z}_{M}^{m}=j\left[\begin{array}{cc}
-\mathbf{X}_{A} & -\sqrt{r_{\mathrm{opt}}} \mathbf{R}_{A}^{1 / 2} \\
-\sqrt{r_{\mathrm{opt}}} \mathbf{R}_{A}^{1 / 2} & x_{\mathrm{opt}} \mathbf{I}_{M}
\end{array}\right]
$$

where $\mathbf{X}_{A}=\operatorname{Im}\left\{\mathbf{Z}_{A}\right\}, r_{\text {opt }}=\operatorname{Re}\left\{z_{\text {opt }}\right\}, x_{\text {opt }}=\operatorname{Im}\left\{z_{\text {opt }}\right\}$, and $\mathbf{R}_{A}=(1 / 2)\left(\mathbf{Z}_{A}^{H}+\mathbf{Z}_{A}\right)$. From (32) and (33), we see that this matching network effectively uncouples the antennas, and so the noise covariance in (33) reduces to spatially white noise, $\mathbf{T}_{a}=N_{0} \mathbf{I}$, where $N_{0}=4 k_{B} T_{0} B F_{\min } r_{\text {opt }}$.

The optimal multiport match can be difficult to realize in practice. We therefore also consider a simpler, suboptimal type of matching, called self-matching $[12,14]$, in which a two-port matching network is connected to each single dipole that achieves the minimum noise figure for that antenna in isolation. A reciprocal and passive network that implements this match is

$$
\mathbf{Z}_{M}^{s}=j\left[\begin{array}{cc}
-\mathbf{X}_{\mathrm{AS}} & -\sqrt{r_{\mathrm{opt}}} \mathbf{R}_{\mathrm{AS}}^{1 / 2} \\
-\sqrt{r_{\mathrm{opt}}} \mathbf{R}_{\mathrm{AS}}^{1 / 2} & x_{\mathrm{opt}} \mathbf{I}_{M}
\end{array}\right]
$$

where $\mathbf{Z}_{\mathrm{AS}}=\operatorname{diag}\left(\mathbf{Z}_{\mathrm{A}}\right), \mathbf{R}_{\mathrm{AS}}=\operatorname{Re}\left\{\mathbf{Z}_{\mathrm{AS}}\right\}$, and $\mathbf{X}_{\mathrm{AS}}=\operatorname{Im}\left\{\mathbf{Z}_{\mathrm{AS}}\right\}$. Here $\operatorname{diag}(\cdot)$ retains only the diagonal entries of the matrix.

In Figure 3, we plot the expected sum-rate (4) versus SNR of coordinated beamforming systems for $M=2$ receive antennas spaced $d=0.1 \lambda$ apart. When optimal multiport matching (38) is used, the noise covariances $\mathbf{T}_{1}$ and $\mathbf{T}_{2}$ are spatially white and the coordinated beamforming algorithm for uncorrelated noise (CB-U) in Section 2.1 can be applied. When perfect CSI is available (CSIT), this algorithm yields the largest sum-rate in Figure 3 for all SNRs. In fact, the sum-rate of this highly coupled system is slightly better than the performance obtained for i.i.d. fading and noise in [8]. Although it may appear surprising 


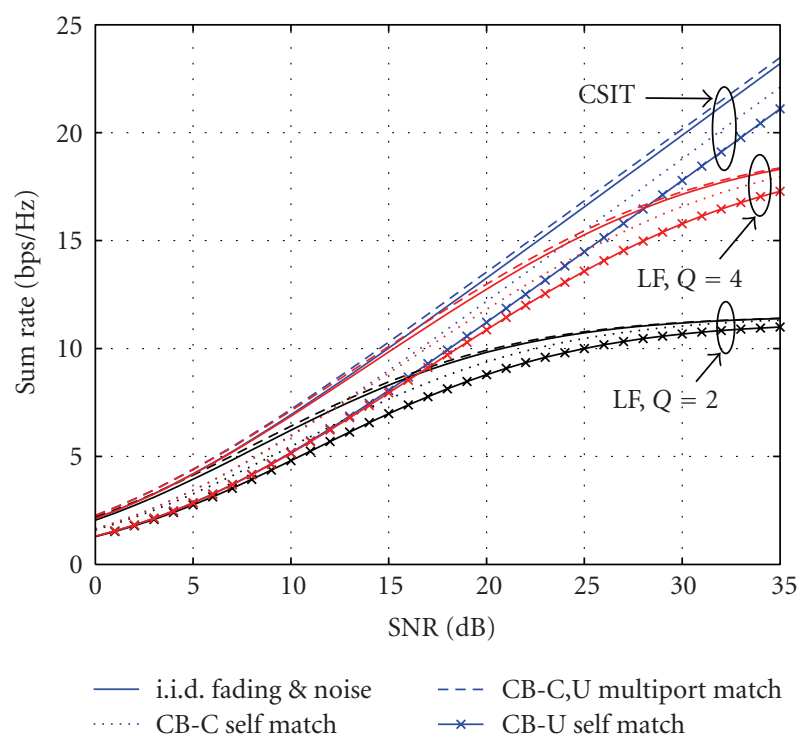

FIgURE 3: Sum-rate versus SNR with $N=M=2$ and $d=0.1 \lambda$.

that a highly coupled system can actually perform slightly better than i.i.d. fading and noise, similar results have been reported for MIMO capacity [19] and receive diversity [14]. (Wallace and Jensen [19] suggested the explanation that closely-spaced antennas can actually collect more power than widely separated ones because part of the power scattered by each receive antenna can be recaptured by the adjacent antenna, especially when appropriate matching is implemented.) Using the simpler but suboptimal self-match (39) with $\mathrm{CB}-\mathrm{U}$ leads to a performance loss of roughly $3.6 \mathrm{~dB}$ at high SNRs, due to the presence of correlated noise in the receivers. If we compensate for the correlation by applying the new coordinated beamforming algorithm for correlated noise (CB-C) in Section 2.2, the loss is reduced to about $1.8 \mathrm{~dB}$. When self-matching is used, note that both algorithms enforce zero multiuser interference at the receivers; however, $\mathrm{CB}-\mathrm{C}$ also exploits the noise correlation present in coupled receivers to further improve performance.

Figure 3 also shows the expected sum-rate of these four systems with limited feedback (LF) with $Q=2$ bits (black lines) or $Q=4$ bits (red lines), as described in Section 2.3. Again we see that CB-U with multiport matching provides the best performance and is slightly better than the performance obtained for i.i.d. fading and noise in [8]. The performance loss entailed by using self-matching with CB- $\mathrm{U}$ is in the range $3.6-8.0 \mathrm{~dB}$ for $Q=2$ and $3.6-5.0 \mathrm{~dB}$ for $Q=4$. If the new algorithm $C B-C$ is used with selfmatching, however, these losses are reduced to $0.8-1.8 \mathrm{~dB}$ for $Q=2$ and $1.0-1.8 \mathrm{~dB}$ for $Q=4$. For SNRs in the range $0-$ $5 \mathrm{~dB}$, note that all of the limited feedback curves are close to the corresponding full CSI curves, so $Q=2$ could be used with little loss of performance. The limited feedback systems with $Q=4$ perform close to full CSI systems for SNRs up to $15 \mathrm{~dB}$. For higher SNRs, a large gap opens up between the performance of the new algorithm with full CSI

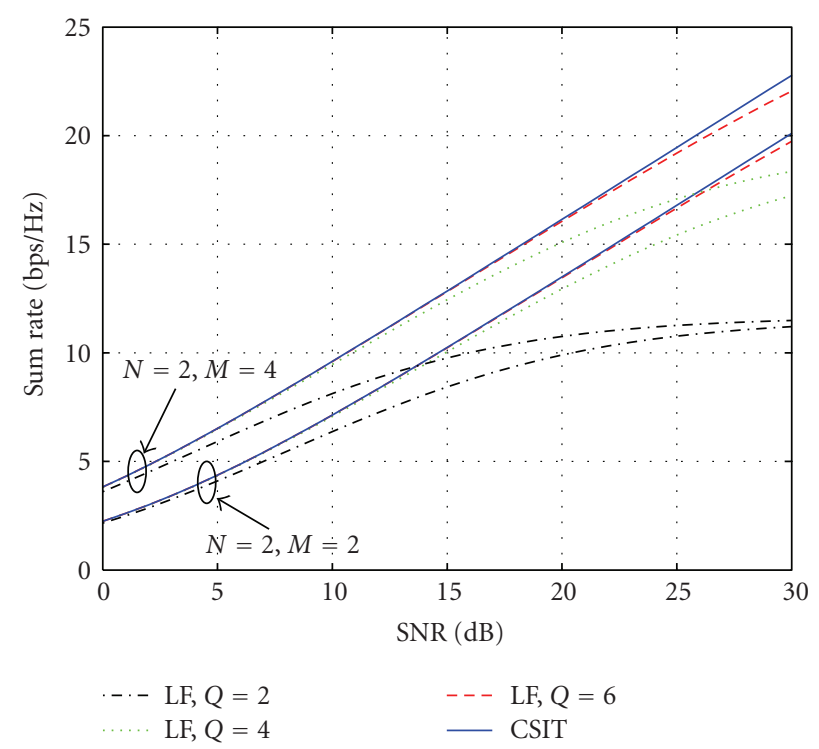

FIGURE 4: Sum-rate for multiport matching versus SNR with $N=2$, $M=2$ or 4 and $d=0.2 \lambda$.

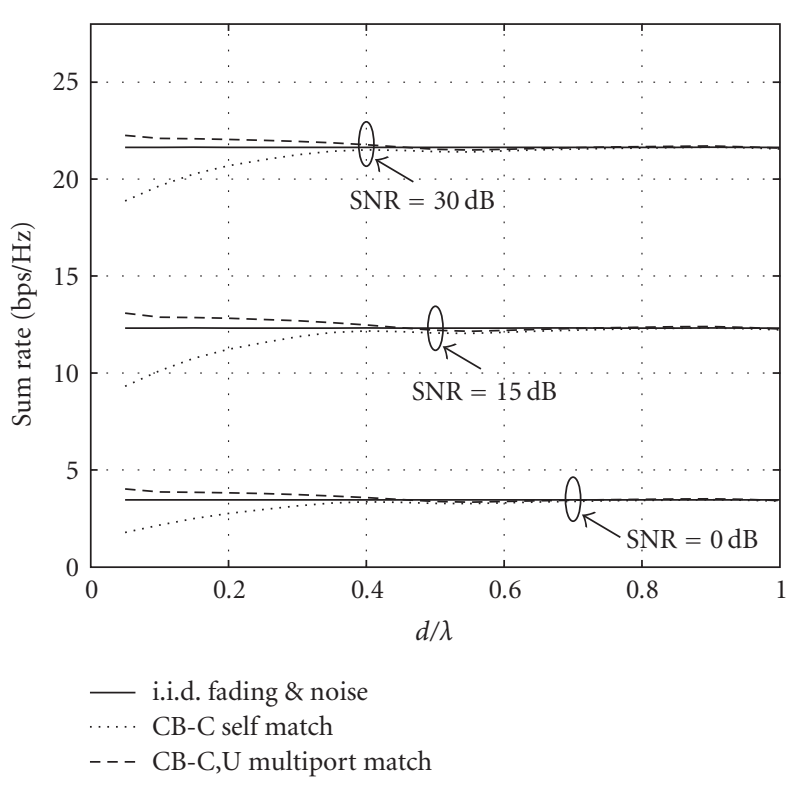

FIGURE 5: Sum-rate versus antenna spacing with $N=2, M=4$ and $Q=6$.

and the limited feedback versions, which might be mitigated by choosing a larger $Q$.

The advantages of larger arrays are illustrated in Figure 4, which plots the expected sum-rate of CB-C (or CB-U) with multiport matching for $M=2$ and 4 receive antennas, spaced $0.2 \lambda$ apart, and different feedback scenarios. For full CSI, the larger array improves the sum-rate by a consistent $4.7 \mathrm{~dB}$ relative to the $M=2$ case. For limited feedback scenarios, however, the improvement decreases as SNR increases, particularly for small $Q$. 

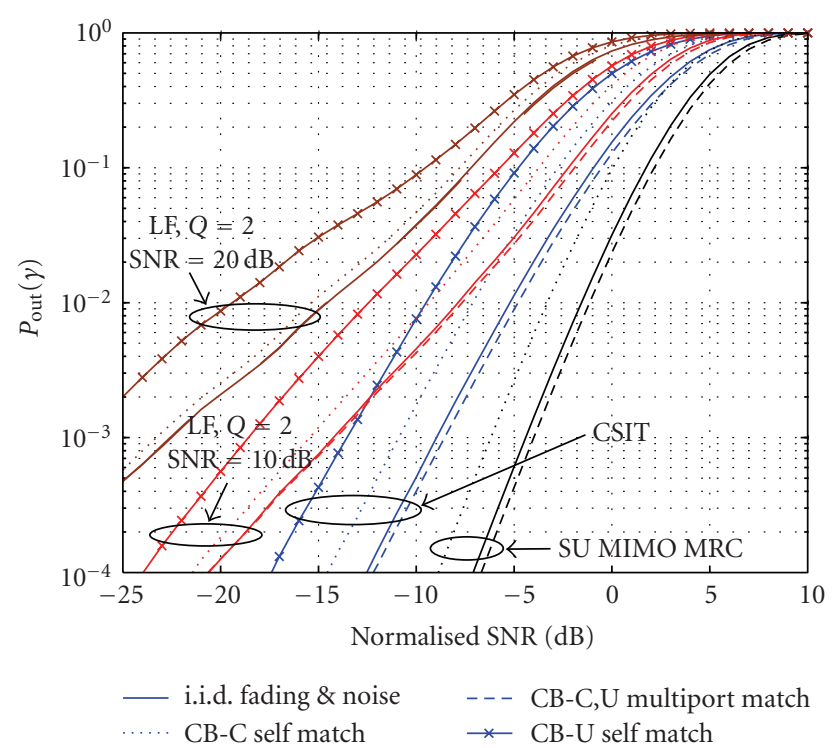

FIgURE 6: Outage probability versus normalized SNR with $N=$ $M=2$ and $d=0.1 \lambda$.

We now consider the impact of receive antenna spacing on the expected sum-rate. Figure 5 plots the expected sumrate of the proposed beamforming system for $M=2$, limited feedback $Q=6$, and three different SNRs $\left(P / N_{0}=\right.$ $0 \mathrm{~dB}, 15 \mathrm{~dB}$, and $30 \mathrm{~dB}$ ). Also shown for comparison is the performance of an i.i.d. system with the same SNRs. Note that multiport matching can achieve performance close to the i.i.d. case even in the presence of strong coupling, whereas performance with self-matching tends to degrade for $d<$ $0.4 \lambda$.

Another performance metric of interest in coordinated beamforming is the outage probability

$$
P_{k}^{\text {out }}(\tau)=\operatorname{Pr}\left\{\gamma_{k}<\tau\right\}
$$

where $\tau$ is a nonnegative threshold and $\gamma_{k}$ is defined by (5) for full CSI systems and by (20) for limited feedback. Since no closed-form formulas exist for the outage probability of coordinated beamforming systems, we will estimate it by Monte Carlo methods.

The outage probabilities versus normalized SNR are shown in Figure 6 for coordinated beamforming systems with $M=2$ antennas spaced $d=0.1 \lambda$ apart. Also shown for comparison are results for a single-user MIMO MRC system with mutual coupling (black lines) from [20]. Here normalized SNR means that $P_{k}^{\text {out }}(\tau)$ is plotted versus

$$
\bar{\tau}_{k}=\frac{\tau}{P_{k} / N_{0}}=\frac{\tau}{P / 2 N_{0}}
$$

where $P_{k} / N_{0}$ is the average SNR of user $k$ with zero multiuser interference. For perfect CSI (blue lines), we see that CB-U (and CB-C) with multiport matching yields the best outage of all the coordinated beamforming systems considered, and

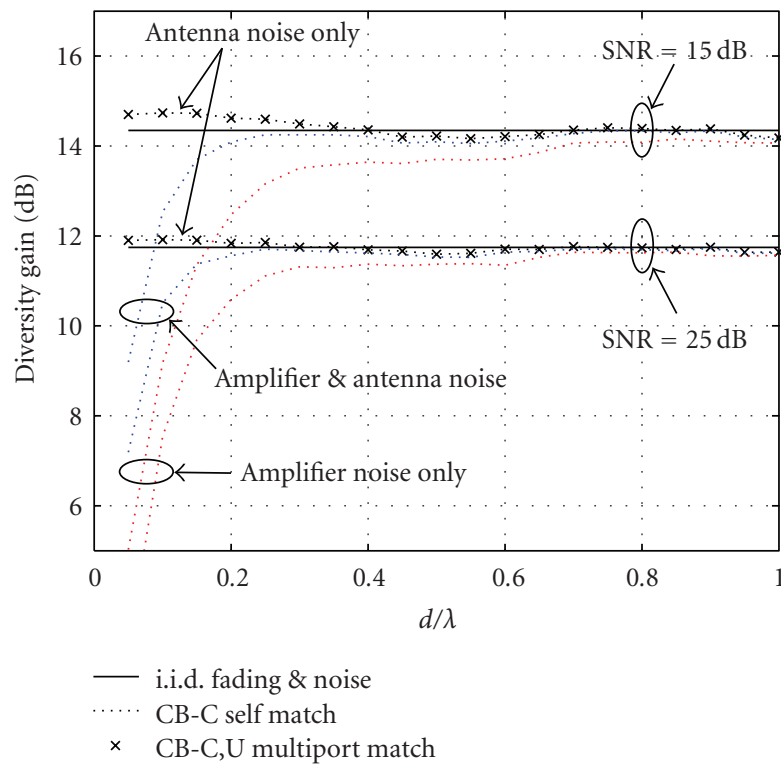

FIGURE 7: Diversity gain versus antenna spacing with $N=M=2$ and $Q=4$ for different noise sources.

at $1 \%$ outage is about $3.7 \mathrm{~dB}$ away from the performance of a single-user MIMO MRC system with mutual coupling (black lines). For self-matching, CB-U suffers a loss of about $5 \mathrm{~dB}$ relative to multiport matching at $1 \%$ outage. If the new algorithm CB-C is used with self-matching, this loss is reduced to $2.3 \mathrm{~dB}$. Figure 6 also plots the outage of limited feedback systems with $Q=2$ bits (red and brown lines). At $1 \%$ outage, the new algorithm CB-C with self-matching performs within $1.7 \mathrm{~dB}$ of $\mathrm{CB}-\mathrm{U}$ with multiport matching for $P / N_{0}=10 \mathrm{~dB}$, and within $0.7 \mathrm{~dB}$ for $P / N_{0}=25 \mathrm{~dB}$. However the algorithm CB-U with self match suffers about $5 \mathrm{~dB}$ loss for both SNRs at the same outage level. Note that LF systems perform poorly for high SNRs because multiuser interference is not completely canceled and the performance becomes interference limited since

$$
\frac{\gamma_{k}}{P / 2 N_{0}}=\frac{\left|\mathbf{g}_{k}^{H} \mathbf{H}_{k} \mathbf{w}_{k}\right|^{2}}{P / 2 N_{0}\left|\mathbf{g}_{k}^{H} \mathbf{H}_{k} \mathbf{w}_{l}\right|^{2}+\left(1 / N_{0}\right) \mathbf{g}_{k}^{H} \mathbf{T}_{k} \mathbf{g}_{k}} .
$$

Further insights can be gained by examining the effect of different noise sources on the diversity gain, which is defined as the difference in SNR between the $M=2$ and $M=1$ outage curves at a fixed outage probability. Figure 7 shows the diversity gain of CB-C at $1 \%$ outage versus antenna spacing $d / \lambda$ for $M=2$, limited feedback $Q=4$, and different types of noise and matching conditions. Note that the diversity gain of CB-C with self-matching decreases as $d / \lambda$ decreases in a way that depends on which source of noise is dominant. When antenna noise is dominant (e.g., $r_{a}=g_{a}=0$ ), the performance of CB-C with self-matching is exactly the same as multiport matching. On the other hand, when amplifier noise dominates (e.g., $\mathbf{T}_{o}=\mathbf{0}$ ) then 
the diversity gain tends to deteriorate rapidly as $d / \lambda$ decreases below 0.2 . This can be explained by observing that antenna noise and the signal enter the receiver at the same point, so that all subsequent impedances transform the signal and noise in the same way, and so do not affect performance. Amplifier noise is more of a problem because noise at the input terminal of amplifier 1 can be transferred via mutual coupling to antenna 2 and become amplified by amplifier 2. Finally, observe that $\mathrm{CB}-\mathrm{C}$ (or $\mathrm{CB}-\mathrm{U}$ ) with multiport matching can achieve a diversity gain close to the i.i.d. case even when the receive antennas are strongly coupled, regardless of what type of noise dominates. For large $d$, note that the antennas become less coupled and the performance of both matching networks converges to the i.i.d. case for each type of noise.

The results above suggest that the performance benefits of coordinated beamforming extend to MIMO broadcast channels with strong correlation and mutual coupling at the receiver. All of the information needed by the transmitter about the noise environment, antennas, matching networks and amplifiers at receiver $k$ can be lumped into a single matrix $\widehat{\mathbf{F}}_{k}$, which is fed back as CSI to the transmitter. Simulations suggest that limited feedback methods can often attain performance comparable to full CSI, although the amount of feedback depends in an essential way on the SNR. Simulations further show that multiport matching is often significantly better than self-matching, particularly in strongly coupled systems. When multiport matching is used, coordinated beamforming can provide performance close to the i.i.d. case even when the receive antennas are spaced as close as $0.2 \lambda-0.4 \lambda$ apart.

\section{Conclusion}

We investigated the effect of receiver correlation, mutual coupling, matching networks, and correlated noise sources on coordinated beamforming systems. We presented a new coordinated beamforming technique for two receivers appropriate for MIMO broadcast channels with signal and noise correlation at the receiver. The best sum-rate and outage performance is attained when optimal multiport matching is used with the CB-U algorithm in [8]. Since multiport matching is difficult to achieve in practice, we also considered the performance of suboptimal self-matching. Numerical results suggest that the proposed CB-C algorithm can significantly outperform CB-U in coupled systems with self-matching. These results also suggest that performance depends on which noise sources are dominant. We conclude that, even in the presence of strong coupling, most of the benefits of coordinated beamforming can be preserved by using appropriate matching networks and linear beamforming. Moreover, these benefits can be achieved even when feedback is limited.

\section{Acknowledgment}

This material is based upon work supported by the National Science Foundation under Grant CCF-0728803.

\section{References}

[1] G. J. Foschini and M. J. Gans, "On limits of wireless communications in a fading environment when using multiple antennas," Wireless Personal Communications, vol. 6, no. 3, pp. 311-335, 1998.

[2] L.-U. Choi and R. D. Murch, "A transmit preprocessing technique for multiuser MIMO systems using a decomposition approach," IEEE Transactions on Wireless Communications, vol. 3, no. 1, pp. 20-24, 2004.

[3] C. B. Peel, B. M. Hochwald, and A. L. Swindlehurst, "A vector-perturbation technique for near capacity multiantenna multiuser communication-part I: channel inversion and regularization," IEEE Transactions on Communications, vol. 53, no. 1, pp. 195-202, 2005.

[4] T. Yoo and A. Goldsmith, "On the optimality of multiantenna broadcast scheduling using zero-forcing beamforming," IEEE Journal on Selected Areas in Communications, vol. 24, no. 3, pp. 528-541, 2006.

[5] K.-K. Wong, "Maximizing the sum-rate and minimizing the sum-power of a broadcast 2-user 2-input multiple-output antenna system using a generalized zeroforcing approach," IEEE Transactions on Wireless Communications, vol. 5, no. 12, pp. 3406-3412, 2006.

[6] D. J. Love and R. W. Heath Jr., "Limited feedback diversity techniques for correlated channels," IEEE Transactions on Vehicular Technology, vol. 55, no. 2, pp. 718-722, 2006.

[7] P. Xia and G. B. Giannakis, "Design and analysis of transmitbeamforming based on limited-rate feedback," IEEE Transactions on Signal Processing, vol. 54, no. 5, pp. 1853-1863, 2006.

[8] C.-B. Chae, D. Mazzarese, N. Jindal, and R. W. Heath Jr., "Coordinated beamforming with limited feedback in the MIMO broadcast channel," IEEE Journal on Selected Areas in Communications, vol. 26, no. 8, pp. 1505-1515, 2008.

[9] T. Svantesson and A. Ranheim, "Mutual coupling effects on the capacity of multielement antenna systems," in Proceedings of the IEEE International Conference on Acoustics, Speech, and Signal Processing (ICASSP '01), vol. 4, pp. 2485-2488, Salt Lake City, Utah, USA, May 2001.

[10] R. Janaswamy, "Effect of element mutual coupling on the capacity of fixed length linear arrays," IEEE Antennas and Wireless Propagation Letters, vol. 1, no. 1, pp. 157-160, 2002.

[11] B. Clerckx, D. Vanhoenacker-Janvier, C. Oestges, and L. Vandendorpe, "Mutual coupling effects on the channel capacity and the space-time processing of MIMO communication systems," in Proceedings of the IEEE International Conference on Communications (ICC '03), vol. 4, pp. 2638-2642, Anchorage, Alaska, USA, May 2003.

[12] B. K. Lau, J. B. Andersen, G. Kristensson, and A. F. Molisch, "Impact of matching network on bandwidth of compact antenna arrays," IEEE Transactions on Antennas and Propagation, vol. 54, no. 11, pp. 3225-3238, 2006.

[13] M. L. Morris and M. A. Jensen, "Improved network analysis of coupled antenna diversity performance," IEEE Transactions on Wireless Communications, vol. 4, no. 4, pp. 1928-1934, 2005.

[14] C. P. Domizioli, B. L. Hughes, K. G. Gard, and G. Lazzi, "Receive diversity revisited: correlation, coupling and noise," in Proceedings of the IEEE Global Telecommunications Conference (GLOBECOM '07), pp. 3601-3606, Washington, DC, USA, November 2007.

[15] C. B. Balanis, Antenna Theory: Analysis and Design, John Wiley \& Sons, New York, NY, USA, 3rd edition, 2005.

[16] H. A. Haus and R. B. Adler, Circuit Theory of Linear Noisy Networks, John Wiley \& Sons, New York, NY, USA, 1959. 
[17] A. Voors, 4nec2, http://home.ict.nl/ arivoors.

[18] http://datasheets.maxim-ic.com/en/ds/MAX2642MAX2643.pdf.

[19] J. W. Wallace and M. A. Jensen, "Mutual coupling in MIMO wireless systems: a rigorous network theory analysis," IEEE Transactions on Wireless Communications, vol. 3, no. 4, pp. 1317-1325, 2004.

[20] Y. Dong, B. L. Hughes, and G. Lazzi, "The impact of mutual coupling on MIMO maximum-ratio combining," in Proceedings of the IEEE Global Telecommunications Conference (GLOBECOM '07), pp. 4516-4521, Washington, DC, USA, November 2007. 